\title{
Neural network and improved method for wind power prediction
}

\author{
Rui $\mathrm{Li}$ \\ Department of Electrical Engineering \\ North China Electric Power University \\ Bao ding, China \\ liruibdwdm@yeah.net
}

\begin{abstract}
A single population genetic algorithm is introduced to optimize the weight value and threshold value of the BP network (SGABP) to overcome the issues of the slowly convergence speed and easy to fall into local optimum of $B P$ neural network. Taking the premature of genetic algorithm (GA) into account, a multi-population genetic algorithm is constructed to optimize BP network prediction model, the MPGABP model improves the SGABP model by adding immigrants operator and artificial selection operator; Besides, we improve the generalization ability of system in the machine learning methods, through using the noise sequence method to avoid over-fitted. By using the above models to wind power prediction, we draw a conclusion that the combination of the artificial intelligence algorithms is more effective than a single prediction method to improve the prediction accuracy.
\end{abstract}

Keywords-Neural network; genetic algorithm; multipopulation genetic algorithm; over-fitted; wind power prediction

\section{INTRODUCTION}

As a new type of power generation following the solar power technology, wind power has achieved rapid development in many countries in recent years.

The feature of randomness and fluctuations restricts wind power to be networked, which leads to a high percentage of abandoned wind phenomenon, caused huge economic losses. Improve the accuracy of wind electric power prediction can effectively promote wind power to be consumed.

Wind power forecast has been a lot of literature to be studied, including physical forecasting methods, statistical forecasting methods, learning prediction method and combination forecasting method. Xiuyuan Yang, Yang Xiao and Shuyong Chen made a research based on time series and neural network to predict the wind speed, and achieved a good accuracy; Jun-fang $\mathrm{Li}$ introduce four gray models to predict the average wind speed in 1 hour intervals, and pointed out that the traditional GM $(1,1)$ model has higher accuracy than other models in terms of achieving a higher forecast of wind power; Jifu Qian proposed a short-term forecasting method based on least squares support vector machine, using historical wind data, pressure, temperature as input, wind and environmental conditions for training, predictive modeling, grid search method to determine the model parameters.
In this article the neural network is introduced to predict the wind power, using Statistical regularities to identify and revise the singular value of original sequence. BP neural network is applied to predict wind power. A single population genetic algorithm is introduced to optimize the weight value and threshold value of the BP network (SGABP) to overcome the issues of the slowly convergence speed and easy to fall into local optimum of BP neural network. Taking the premature of genetic algorithm (GA) into account, a multi-population genetic algorithm is constructed to to optimize BP network prediction model, the MPGABP model improves the SGABP model by adding immigrants operator and artificial selection operator; Besides, we improve the generalization ability of system in the machine learning methods, through using the noise sequence method to avoid over-fitted.

\section{BP NETWORK ALGORITHM}

BP neural network is one of the most widely used network model in the study of neural network, the use of negative gradient descent algorithm, effectively overcome some of the problems which the single-layer perceptron can not solve. BP neural network which contains only a input layer, a hidden layer and output layer, has a strong approximation ability [4][5] and is widely used in forecasting, pattern recognition, signal processing and many other fields.

\section{A. BP network algorithm}

BP (Back Propagation) algorithm is based on the minimum mean square error learning rule. Provide a suitable sample set $\left\{x_{1}, t_{1}\right\},\left\{x_{2}, t_{2}\right\}, \ldots,\left\{x_{q}, t_{q}\right\}$ to the network, where ${ }^{x_{q}}$ indicates the input of the network, ${ }^{t}$ represents of the corresponding correct target output. Using the minimum mean square error between the actual output $a_{q}$ and the target output $t_{q}$ of the network to adjust the weights value and thresholds of network. As shown in the following formula: 


$$
\begin{aligned}
& a=f\left(\sum_{i=1}^{q} W i X i+b i\right) \\
& a^{m+1}=f^{m+1}\left(W^{m+1} a^{m}+b^{m}\right), m=1,2, \ldots, M-1 \\
& a^{1}=x \\
& \begin{array}{l}
a=a^{M} \\
\text { Approximate gradient descent algorithm can be }
\end{array}
\end{aligned}
$$
expressed as:

$$
\begin{gathered}
w_{i j}^{m}(k+1)=w_{i j}^{m}(k)-\alpha \frac{\partial F}{\partial n_{i}^{m}} a_{j}^{m-1} \\
b_{i}^{m}(k+1)=b_{i}^{m}(k)-\alpha \frac{\partial F}{\partial b_{i}^{m}}
\end{gathered}
$$

where

$$
\frac{\partial F}{\partial n^{m}}=\left[\begin{array}{l}
\frac{\partial F}{\partial n^{m}} \\
\frac{\partial F}{\partial n^{m}} \\
\cdots \\
\frac{\partial F}{\partial n^{m}}
\end{array}\right]
$$

\section{B. BP neural network deficiencies}

A network model can be evaluated in terms of computing time, the complexity of network topology, the efficiency of the algorithm. Based on above mathematical theory analysis, one conclusion can be draw that BP neural network has a more rigorous theoretical support, forward conduction of signals in the network and error back propagation is very clear. However, due to the BP neural network is a network model based on the negative gradient descent algorithm, inevitably, there are some disadvantages:

(1) Cannot escape from the local minimum. BP learning rule which based on the negative gradient descent method is to calculate and minimize the mean square error. Gradient descent process is likely to fall into a local minimum value and could not get out of the shock phenomenon as a result of error surface has many minimum points.

(2) A long time to learn and slow convergence rate. The shock phenomenon will lead to BP neural network to increase learning time and slow down the convergence rate in the training process, In extreme cases, may also lead to the network does not converge. Besides, weight adjustment in the training process may also let the activation function come to a situation of saturation, correspondingly the adjustments of the value of the right almost at a standstill status, thus extending the training time.

\section{Improved BP Neural Network technology}

- $\quad$ additional momentum BP algorithm
Additional momentum BP algorithm using a momentum factor $(0<\mathrm{z}<1)$ based on BP algorithm, the expression can be shown as follows:

$$
\begin{gathered}
\Delta W^{m}(k+1)=\gamma \Delta W^{m}(k)-(1-\gamma) \partial \frac{\partial F}{\partial W^{m}} \\
W^{m}(k+1)=W^{m}(k)+\Delta W^{m}(k+1)
\end{gathered}
$$

\section{- $\quad$ variable learning rate $\mathrm{BP}$ algorithm}

In the traditional BP algorithm and additional momentum BP algorithm, the learning rate of weights and thresholds are fixed. The choice of the learning rate is a more sensitive issue for the learning algorithm, If the learning rate is too large, the algorithm may produce shock; if learning rate is too small, it is possible to make the speed of algorithm is too slow to convergence. The main idea of the variable learning rate $\mathrm{BP}$ algorithm is:

When concentrated in the entire sample, the mean square error decreases gradually, weights and threshold correction is in the right direction, then you can increase the learning rate, the learning rate $\alpha$ is multiplied by the incremental factor $\eta$; When the mean square error increases and exceeds a certain value, the correction is in the wrong direction, the learning rate $\alpha$ should be reduced, so that the learning rate $\alpha$ multiplied by the reduction factor $\rho$.

Learning rate correction can be shown as follows:

$$
\begin{aligned}
& \alpha(k+1)=\eta \alpha(k), E(k+1) \leq E(k) \\
& \alpha(k+1)=\rho \alpha(k), E(k+1)>E(k)
\end{aligned}
$$

\section{- $\quad$ intelligent optimization technology}

Currently, there are many ways of using artificial intelligence optimization method, Such as: Holland proposed genetic algorithm (Genetic Algorithm), [6] Kirkpatrick proposed simulated annealing algorithm (Simulated Annealing) [7], Dorigo introduced the ant colony algorithm (Ante Colony optimization) [8], particle swarm optimization (Particle Swarm Optimization) proposed by Kennedy and Eberhar [9] and so on.

\section{GENETIC ALGORITHM}

In the above-described optimization methods, the combination of neural network and genetic algorithm is more well-known. The genetic algorithm consists of the following components:

\section{A. chromosome coding}

The parameters of practical problems can be mapped to individual chromosomes by encoding in the genetic algorithm. Coding problem is of great value for the search capabilities of the algorithm and the diversity of the population. Originally coded by binary form which is composed by a string of digits 0 and 1 , the main advantage is the mapping algorithm in the encoding and decoding process is simple, easy to implement. 


\section{B. Selection Operator}

Selection operator is used to eliminate maladjusted individual and retain high-quality individuals based on the assessment of the fitness of each chromosome to adapt to the environment. The commonly used method of selecting can be described as the following categories: roulette wheel selection, ranking selection method, the expected value method.

\section{C. crossover operator}

The crossover operator is to select two individuals from the population, through the combination of two individual chromosome genetic exchange to generate new individuals. During the cross process, random selection of one, two, or with consistent cross strategy are allowed. By using crossover operator in the Genetic Algorithms, the information of individual chromosomes in the population can be exchanged, which may result in the quality of individual which constitutes a new point in the search space, the search ability of the genetic algorithm has been significantly improved.

\section{The mutation operator}

The mutation operator is to choose an individual from the population to change the gene of chromosomal on one location to produce a new individual. The mutation operator makes some location of the gene of a single individual chromosome for random change, which not only improves the local random search capabilities, but also maintains the diversity of the population.

\section{E. fitness function}

In the genetic algorithm, the fitness function is used as a standard to assess the merits of individual of the population. The fitness function plays an important role in affecting the performance of the algorithm. In practice, the objective function of the problem is directly mapped into a fitness function, thus individual fitness value can be calculated by the fitness function, available to genetic manipulation and zoom fitness function can effectively control the strength of selection operator in genetic operator.

\section{GENETIC ALGORITHM TO OPTIMIZE BP}

When using the genetic algorithm to improve BP neural network, at first the weights and threshold values of $\mathrm{BP}$ neural network are mapped to individual chromosomes of the population, then using floating-point numbers to encode the individual, collection of populations which consists of weights and thresholds repeated iterations until the termination condition is reached through genetic manipulation. Thus we obtained a more excellent weights and threshold values, and then give it to the neural network, which solves the problem of randomly selected value and the threshold value of the BP neural network.

Using genetic algorithm to optimize BP neural network, can follow these steps:

(1) Set the BP neural network structure, initialize the network weights and threshold, converted it into individual chromosome of genetic algorithm;
(2)Initialize parameters of genetic algorithm including population size, crossover probability and mutation probability and the number of iterations;

(3)Evaluate the fitness of each individual in the population;

(4)Genetic manipulation including selection operation, crossover and mutation;

(5)If the results do not meet the conditions, return to the previous step. Otherwise, let the optimal results back to the BP neural network as the initial weights.

(6)BP neural network experiment, get the conclusion.

A single population genetic algorithm is introduced to optimize the weight value and threshold value of the BP network (SGABP) to overcome the issues of the slowly convergence speed and easy to fall into local optimum of BP neural network.

\section{MULTI-POPULATION GENETIC ALGORITHM TO OPTIMIZE BP NEURAL NETWORK}

Premature convergence phenomenon can not be ignored in the genetic algorithm, mainly manifested in all individuals in the population tends to be the same and stop the evolution, as a result the algorithm cannot give a satisfactory solution ultimately.

To improve the deficiency of GA, a variety of population genetic algorithm (multiple population GA, MPGA) can be used to instead the conventional standard genetic algorithm (SGA).

Through using multiple populations with different control parameters, MPGA taking the global search and local search into account, makes up the balance ability of global search and local search caused by the value of single for SGA crossover probability Pc and mutation probability Pm. Various groups are independent in SGA model. Through the use of immigration in MPGA, the best individual in the source populations is used to instead the worst individual in the target population, thus contact the various groups.

Taking the premature of genetic algorithm (GA) into account, a multi-population genetic algorithm is constructed to optimize BP network prediction model, the MPGABP model improves the SGABP model by adding the immigrant operator and artificial selection operator.

\section{MACHINE LEARNING SYSTEM GENERALIZATION ABILITY TO IMPROVE}

BP neural network training the network through the learning of existing data, as a result the nonlinear model is base on the existing data, when it is used to forecast the unknown input, due to no previous similar data, the network has a lower recognition ability to the unknown input data, which reduces the generalization performance of the system. In order to prevent the neural network model over-fitted for the training set, add white noise in the original training samples to train the network.

\section{NUMERICAL EXAMPLE}

Using the wind electric power data of January 1998 of one certain district to analysis the above three models. To 
make the input information of the network contains more information of wind power, we use 3 days of wind electric power data as a network input samples, the fourth day of wind electric power data as the output of network, to build a total of 28 samples. Select the top 27 as historical data, to train the neural network. The last sample is used to test the prediction accuracy of the network. Using BP neural network, SGABP network, MPGABP network three ways to predict the wind power, results and error can be show in tablel.

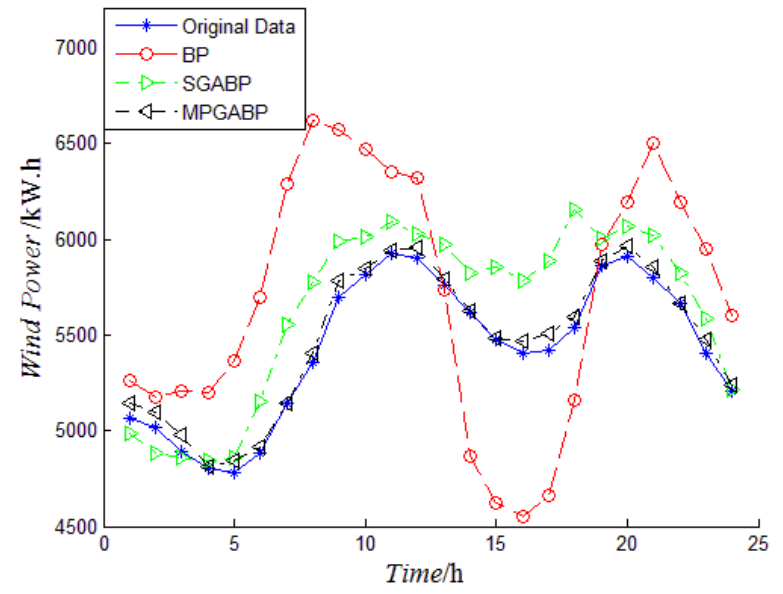

Figure 1. Forecast results

The results is also described in Figure1 and Figure 2.

We can see from Table 1 and Figures 1,2,through a stepby-step improvement, the maximum prediction error ranges from $23.6 \%$ of the BP network, reduced to $11.1 \%$ of the SGABP network, to less than $2 \%$ of the MPGABP network, indicating that the three prediction methods based on artificial intelligence has a better prediction accuracy for the wind power prediction.

Comparing three methods listed above and through the above analysis, a conclusion can be drawn that BP model is simple and easy to implement, but has a relative lower prediction accuracy. The accuracy of SGABP model is improved compared with BP model, but the time complexity of SGABP is relative high. The MPGABP model has the highest prediction accuracy, but takes a long time, that is to say, the time complexity of MPGABP model is very high. Specific practical application should take the time complexity of one method, the prediction accuracy of it and performance indicators into account to assess a particular algorithm.

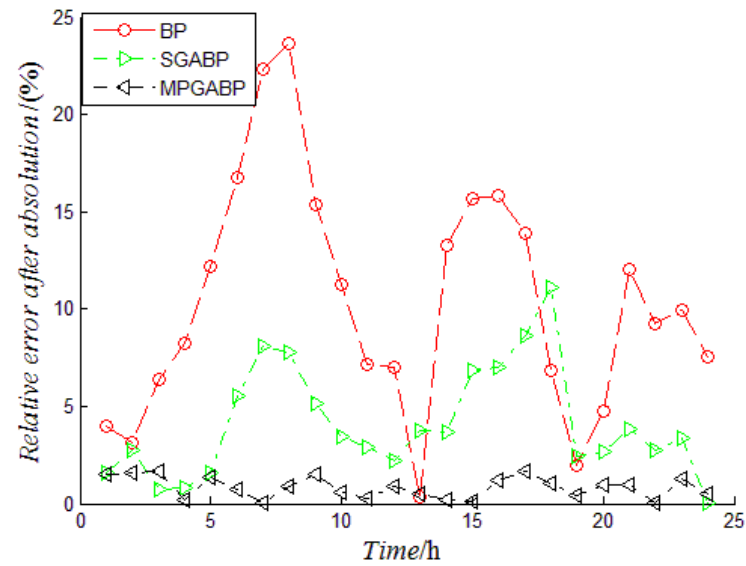

Figure 2. prediction error

\section{SUMMARY}

Artificial intelligence including neural networks, genetic algorithms can be applied to many practical problems. In this article artificial intelligence algorithms are used to wind power prediction. BP model, SGABP model and MPGABP model is introduced step by step to forecast wind power. Adding noise can prevent over-fitted of the network, which improves the generalization ability of the system.

BP neural network is applied to predict wind power. A single population genetic algorithm is introduced to optimize the weight value and threshold value of the BP network (SGABP) to overcome the issues of the slowly convergence speed and easy to fall into local optimum of BP neural network. Taking the premature of genetic algorithm (GA) into account, a multi-population genetic algorithm is constructed to optimize BP network prediction model, the MPGABP model improves the SGABP model by adding immigrants operator and artificial selection operator; Besides, we improve the generalization ability of system in the machine learning methods, through using the noise sequence method to avoid over-fitted.

Artificial intelligence is of great value for wind power prediction and other areas.

\section{REFERENCES}

[1] Xiuyuan Yang, Yang Xiao and Shuyong Chen. Wind speed and power generation forecast [J]. China Proceedings of the CSEE, 2005,25 (11) :1-5.O

[2] Buhan Zhang, Guanglong Xie. Based gray model wind - wind power prediction [J]. Power System Protection and Control, 2010,38 (19) :151-160.

[3] Jifu Qian. SVR seasonal time series prediction model and application of [D]. Guangzhou: South China University of Technology, 2010.

[4] CotterN, Thestone-weierstrass theorem and its applications to neural network [J]. IEEE Transaction on Neural Networks, 1990, 1 (4) : 290-295

[5] Ito Y. Representation of functions by superposition of astepor sigmoidal functions and their Application to neural network theory [J]. Neural Networks, 1991,4 (3) :385-394 
[6] Minqiang Li, Jisong Kou, Lin Dan, Shu Chun Li the basic theory and application of genetic algorithm [M]Beijing: Science Press,2004,1745.

[7] KirkPatrick S, Gelatt CD, Vecchi MP. Optimization by simulated annealing. Science, 1983,220 (4598):671-680
[8] Bonabeaue Dorigo M, Theranlaz G. Inspiration for optimization from social insect behavior [J]. Nature, 2000,406 (6) :39-42

[9] EI-Gallad Al, El-Hawary ME, Sallam A. Swam of intelligent Particles for solving the nonlinear constrained optimization problem $[J]$. Engineering intelligent systems for Electrical engineering and communications, 2001,9 (3) :155-163

TABLE I. SHORT-TERM WIND POWER PREDICTION RESULTS ANALYSIS AND ERROR

\begin{tabular}{|c|c|c|c|c|c|c|c|c|c|c|}
\hline \multirow[b]{2}{*}{ Time } & \multirow[b]{2}{*}{$\begin{array}{c}\text { The } \\
\text { true } \\
\text { value }\end{array}$} & \multicolumn{3}{|c|}{ Forecast results } & \multicolumn{3}{|c|}{ Absolute error } & \multicolumn{3}{|c|}{ Relative error $(\%)$} \\
\hline & & BP & SGABP & MPGABP & BP & SGABP & MPGABP & BP & SGABP & MPGABP \\
\hline $1: 00$ & 5064 & 5264.560825 & 4982. 90219 & 5141.679517 & 200.5608251 & 81. 09781019 & 77. 6795171 & 3. 9605218 & 1.6014575 & 1. 5339557 \\
\hline $2: 00$ & 5019 & 5175.689908 & 4881.522464 & 5098. 613852 & 156.6899078 & 137. 4775357 & 79. 61385248 & 3. 1219348 & 2. 739142 & 1. 5862493 \\
\hline $3: 00$ & 4892 & 5204.65814 & 4855. 493047 & 4974. 715833 & 312.6581404 & 36.5069526 & 82.71583328 & 6.391213 & 0.7462582 & 1. 6908388 \\
\hline $4: 00$ & 4805 & 5199. 815501 & 4842.759389 & 4813. 598267 & 394. 8155014 & 37. 75938866 & 8. 598267042 & 8. 2167638 & 0.7858354 & 0.1789442 \\
\hline $5: 00$ & 4782 & 5363. 90042 & 4857. 306836 & 4845. 816731 & 581. 9004202 & 75. 30683608 & 63.81673096 & 12. 1685575 & 1. 5747979 & 1. 3345197 \\
\hline $6: 00$ & 4880 & 5698.991288 & 5149. 13432 & 4916. 283454 & 818.9912883 & 269. 1343201 & 36.28345394 & 16. 7826084 & 5.5150475 & 0.7435134 \\
\hline $7: 00$ & 5140 & 6289 & 5554.928083 & 5143. 702673 & 1149 & 414. 9280834 & 3. 702673118 & 22. 3540856 & 8. 0725308 & 0.072036 \\
\hline $8: 00$ & 5355 & 6619. 999972 & 5771.310804 & 5404. 239888 & 1264. 999972 & 416. 3108042 & 49. 23988759 & 23. 6227819 & 7. 7742447 & 0.9195124 \\
\hline $9: 00$ & 5696 & 6569. 99974 & 5989. 601639 & 5780.500446 & 873. 9997401 & 293. 6016394 & 84.50044576 & 15. 3440966 & 5. 1545232 & 1. 483505 \\
\hline $10: 00$ & 5815 & 6468.984785 & 6014. 163396 & 5846. 926121 & 653.9847852 & 199. 1633961 & 31.92612085 & 11. 2465139 & 3. 4249939 & 0.5490305 \\
\hline $11: 00$ & 5923 & 6348.685813 & 6093.926251 & 5940. 373956 & 425.6858126 & 170.9262506 & 17. 37395594 & 7. 1869967 & 2. 8858053 & 0.2933303 \\
\hline $12: 00$ & 5901 & 6315.81414 & 6029.919758 & 5953. 246284 & 414.8141403 & 128. 9197582 & 52.24628385 & 7. 0295567 & 2. 1847104 & 0.8853802 \\
\hline $13: 00$ & 5759 & 5737.751699 & 5974. 554778 & 5789. 020653 & 21. 24830054 & 215.5547781 & 30. 0206526 & 0.3689582 & 3. 7429203 & 0.5212824 \\
\hline $14: 00$ & 5616 & 4870.684784 & 5822.416123 & 5626.533746 & 745. 3152159 & 206. 4161232 & 10.53374576 & 13. 2712823 & 3. 6755008 & 0.1875667 \\
\hline $15: 00$ & 5478 & 4620.952684 & 5852.985205 & 5485. 300163 & 857.0473165 & 374.9852046 & 7. 300162861 & 15. 6452595 & 6.845294 & 0.1332633 \\
\hline $16: 00$ & 5406 & 4550.083156 & 5785.623491 & 5469. 308541 & 855.916844 & 379.6234915 & 63. 3085405 & 15.83272 & 7. 0222621 & 1. 1710792 \\
\hline $17: 00$ & 5416 & 4662.738225 & 5882.250793 & 5504.275737 & 753. 2617752 & 466. 250793 & 88.27573703 & 13. 908083 & 8.6087665 & 1. 6299065 \\
\hline $18: 00$ & 5539 & 5158.875722 & 6155.689406 & 5595.889487 & 380. 1242778 & 616.6894064 & 56. 88948732 & 6.8626878 & 11. 1335874 & 1. 0270714 \\
\hline $19: 00$ & 5861 & 5975. 149752 & 6002.8902 & 5886. 458666 & 114. 1497519 & 141. 8901999 & 25. 4586659 & 1. 9476156 & 2. 4209213 & 0.4343741 \\
\hline $20: 00$ & 5910 & 6193.286533 & 6065.62669 & 5965. 271417 & 283. 2865335 & 155. 6266904 & 55.27141731 & 4. 7933424 & 2. 6332773 & 0.9352186 \\
\hline $21: 00$ & 5801 & 6499 & 6023.011552 & 5855.516355 & 698 & 222. 0115519 & 54.51635506 & 12. 0324082 & 3. 8271255 & 0.9397751 \\
\hline $22: 00$ & 5664 & 6189.479794 & 5819. 040855 & 5666. 170104 & 525. 4797941 & 155. 0408551 & 2. 170103791 & 9. 2775387 & 2. 7373032 & 0.038314 \\
\hline $23: 00$ & 5407 & 5944. 999329 & 5587.816574 & 5475.446897 & 537. 9993292 & 180. 8165741 & 68. 44689656 & 9.9500523 & 3. 3441201 & 1. 2658941 \\
\hline $24: 00$ & 5209 & 5603. 983608 & 5211. 768429 & 5234.276637 & 394. 9836078 & 2. 768429256 & 25. 27663669 & 7. 5827147 & 0.053147 & 0.4852493 \\
\hline
\end{tabular}

